\title{
Melting and Crystallization of Ultra-High Molecular Weight Polyethylene with Appearance of Hexagonal Phase II. Strain-Induced Crystallization of Thermal-Contracted Sample
}

\author{
Shinsuke Tsubakinara and Munehisa Yasuniwa \\ Department of Applied Physics, Faculty of Science, Fukuoka University, \\ Jonan-ku, Fukuoka 814-80, Japan
}

(Received September 8, 1995)

\begin{abstract}
Strain-induced crystallization of a thermal-contracted sample, prepared from highly-drawn ultra-high molecular weight polyethylene (UHMW-PE), was examined by the wide angle X-ray diffraction method using a high temperature elongating apparatus. X-Ray diffraction measurements were isothermally carried out at three characteristic temperatures 148 , 152 , and $156^{\circ} \mathrm{C}$, above the melting point. At each temperature, the molten sample was elongated in steps up to the break ratio, and change of the $\mathrm{X}$-ray equatorial diffraction pattern was recorded with a position sensitive proportional counter. At $148^{\circ} \mathrm{C}$ below the orthorhombic to hexagonal transition point determined by thermal analysis of a constrained sample, the strain-induced crystallization of the only orthorhombic crystals occurred in the elongation. On the other hand, the hexagonal crystals grew together with the orthorhombic ones at $152^{\circ} \mathrm{C}$ that corresponds to the transition point, and at a yet higher temperature of $156^{\circ} \mathrm{C}$, the only hexagonal ones grew. Such temperature dependence of the structure of growing crystals was discussed on the basis of the free energy diagram proposed in Part I to explain the melting process of the constrained UHMW-PE.

KEY WORDS Polyethylene / Ultra-High Molecular Weight / Highly-Drawn / Thermal-Contracted / Strain-Induced Crystallization / Orthorhombic / Hexagonal /
\end{abstract}

In the preceding paper (part I), ${ }^{1}$ it was shown that constrained fibers made from ultra-high molecular weight polyethylene (UHMW-PE) melt through an exceptional process accompanied by a transition from the orthorhombic phase to the hexagonal one, and the melting mechanism was explained based on a proposed free energy diagram. On the other hand, if the fibers or highly-drawn samples melt under the unconstrained state, they melt at a melting point of the unconstrained orthorhombic phase without taking the hexagonal phase and contract considerably because highly-oriented molecular chains in crystals change to a more random conformation in the molten state. However, the contracted, i.e., molten sample hardly flows due to its high melt-viscosity attributed to a number of entanglements among the molecular chains, and can be elongated uniformly like a rubber sample by a very weak elongating force. In this elongating process, it is presumed that strain-induced crystallization like that observed in a natural rubber may occur owing to molecular orientation in the direction of elongation.

The crystallization of PE from the stressed molten state $^{2-10}$ or solution ${ }^{11-13}$ has been adequately studied. It was found that the crystal morphologies such as socalled row structure and shish-kebobs were formed through these processes, respectively. Further, there are a few reports of Sakami et al. as to strain-induced crystallization that proceeds isothermally during the elongation of molten samples..$^{14-16}$ However, no study has been reported on such a crystallization process examined directly by X-ray method. If a thermal-contracted sample obtained from the fiber or highly drawn sample made from UHMW-PE is used, a reliable study about the strain-induced crystallization of PE becomes possible by X-ray method because it can be elongated uniformly from the molten state. This study is significant since it may give new fundamental information on the crystallization of polymers and extend the application of PE fibers at elevated temperatures as described in Part I. ${ }^{1}$

In this study (Part II), the strain-induced crystallization of UHMW-PE was investigated for the contracted sample prepared from sample-4 introduced in Part I. For this study, a high temperature elongating apparatus for X-ray measurements was made. At three temperatures above the melting point, changes of the X-ray diffraction pattern in isothermal elongating processes were recorded using it and a position sensitive proportional counter (PSPC). The experimental results are discussed on the basis of the free energy diagram proposed in Part I.

\section{EXPERIMENTAL}

Sample-4 introduced in Part I was used as a highlydrawn UHMW-PE sample. The preparation method was as follows. First, an as-polymerized UHMW-PE powder resin $\left(M_{v}=2.5 \times 10^{6}\right)$ supplied by Showa Denko Co. was dissolved in $p$-xylene at about $140^{\circ} \mathrm{C}$ to a concentration of $0.4 \mathrm{wt} \%$, and then the solution was cooled to room temperature in order to make PE gel come out of it. It was poured into a steel container and dried at $50^{\circ} \mathrm{C}$ in the atmosphere to obtain a PE film. From this film, rectangular strips $5 \mathrm{~mm}$ long by $3 \mathrm{~mm}$ wide were cut out, and manually drawn in a thermo-regulated silicone-oil bath at $135^{\circ} \mathrm{C}$ to an elongation ratio of ca. 60. The obtained samples had the appearance of tape.

Highly-drawn sample-4 was then contracted slowly at $148^{\circ} \mathrm{C}$ in the silicone-oil bath, and cooled to room temperature in atmosphere after taking out from the bath. The sample was contracted to about one third of the original length. Then the contracted sample was immersed into acetone to remove the absorbed siliconeoil and dried in atmosphere. Its shape was rectangular, 


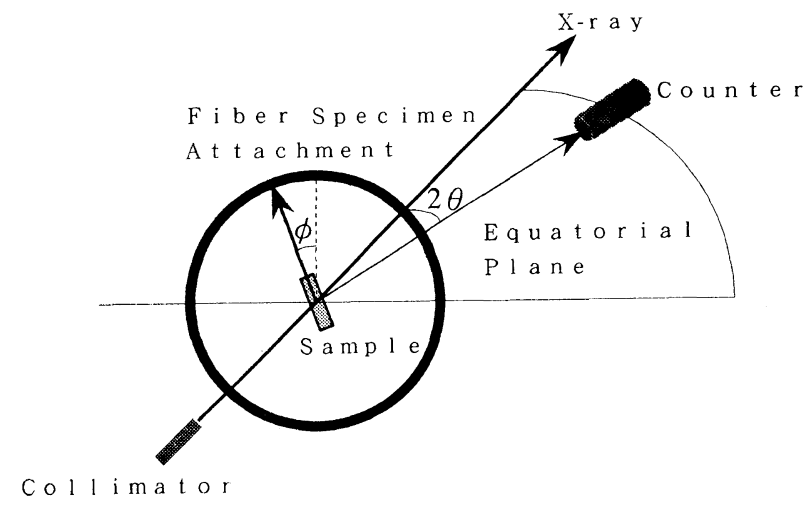

Figure 1. Geometry of X-ray measuring system to examine orientation distribution of $c$-axis. The sample was rotated around the incident beam, and intensity changes of orthorhombic reflections were recorded with the counter fixed at the corresponding diffraction angles.

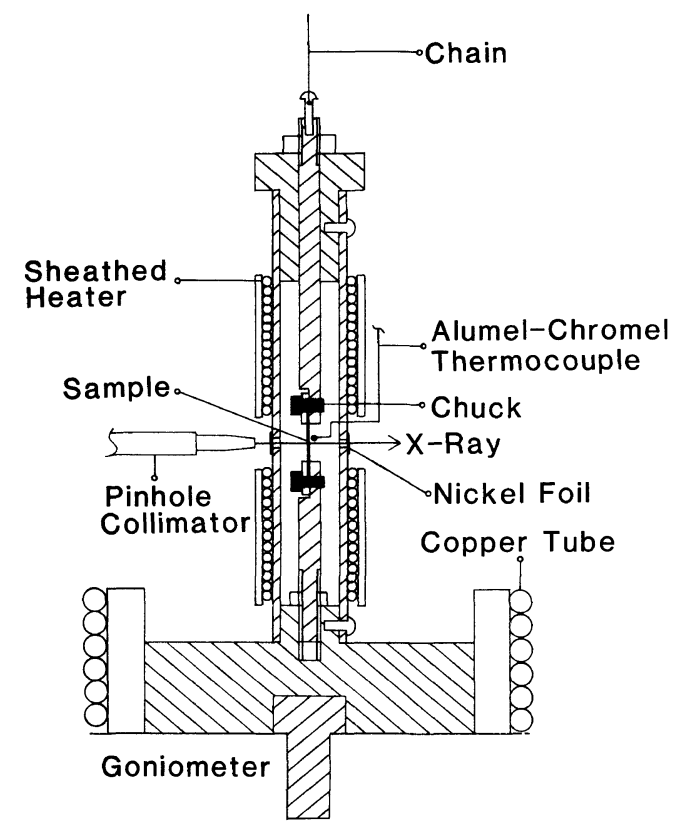

Figure 2. High temperature elongating apparatus for X-ray measurements, used to examine the strain-induced crystallization of the contracted sample. The Ni-foil windows also act as filters for the $\mathrm{Cu}-K_{\beta}$ radiation.

and its dimensions were $c a .0 .7 \mathrm{~mm}$ in width and $c a$. $0.3 \mathrm{~mm}$ in thickness.

For original sample- 4 and the contracted sample, the directional distribution of the $c$-axis corresponding to the molecular axis in crystal was examined at room temperature by X-ray method. The geometry of the measuring system is shown in Figure 1. With the fiber specimen attachment (Rigaku Denki Co.), the sample was rotated around the incident beam $\left(\mathrm{Cu}-K_{\alpha}\right)$ irradiated perpendicularly to the fiber axis, and intensity changes of (110)o and (200)o reflections were recorded as a function of rotation angle $\Phi$ with the counter fixed at the respective diffraction angles on the equatorial plane.

Figure 2 shows a high temperature elongating apparatus for X-ray measurements, made of metallic material such as copper, brass, and stainless steel. It was heated by making an electric current flow through the sheathed heater, and the temperature was precisely controlled with an automatic temperature controller and a cooling circulator that makes a cooled liquid

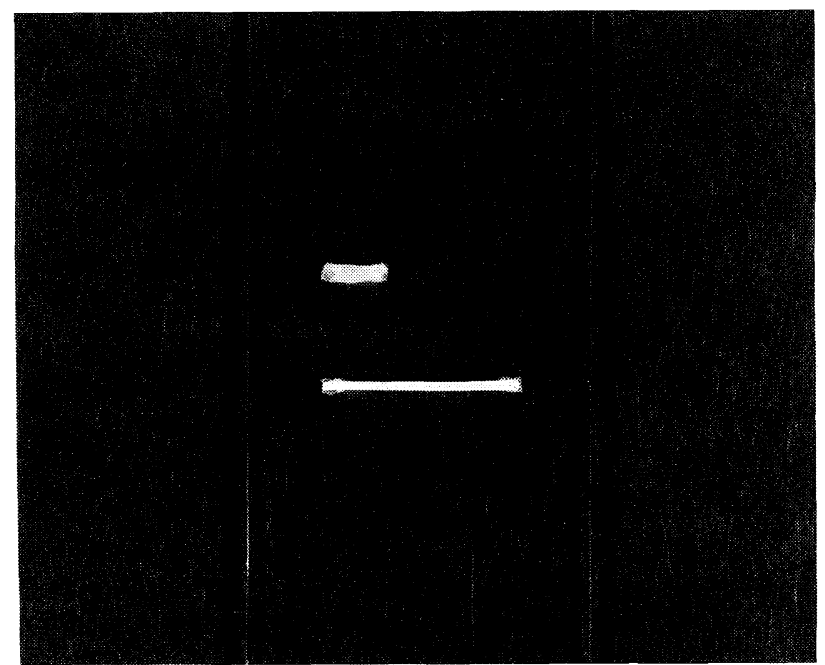

Figure 3. Photograph showing samples contracted (A) and reelongated (B) at $148^{\circ} \mathrm{C}$, obtained from highly-drawn sample-4. The photograph was taken at room temperature.

medium flow through the copper tube. The temperature of the sample was measured by the alumel-chromel thermocouple located in close vicinity to the center of $\mathrm{X}$-ray exposure part. $\mathrm{Cu}-K_{\alpha} \mathrm{X}$-ray radiation was used. The Ni-foil windows acted as filters for the radiation, that is, a pinhole-collimated incident beam of $2 \mathrm{~mm}$ diameter and the diffracted radiation passed through the windows, and $\mathrm{Cu}-K_{\beta}$ radiation was eliminated. The apparatus was mounted on a goniometer, and Rigaku Rotaflex RU-200 $(60 \mathrm{kV}, 200 \mathrm{~mA})$ was used as the X-ray generator.

The contracted sample was set between the two chucks at room temperature, and the apparatus was heated to a desired temperature above the melting point. After thermal equilibration, the sample was elongated in steps up to the break ratio. In this elongating process, the $\mathrm{X}$-ray beam was irradiated to the sample perpendicularly to the fiber axis and its equatorial diffraction pattern was recorded at each elongation ratio with the PSPC detector. About 100 seconds were necessary to the measurement at each ratio. The isothermal elongation was carried out at three characteristic temperatures, 148, 152 , and $156^{\circ} \mathrm{C}$, selected based on the DSC thermogram measured with the constrained sample.

\section{RESULTS AND DISCUSSION}

In this work, fiber samples were not used because the bundled samples could not be contracted uniformly due to differences in contraction ratios among constituent thin filaments. Therefore, highly-drawn sample4 that could be thermally contracted uniformly was selected. Figure 3 is a photograph showing samples that were contracted (A) and re-elongated (B) at $148^{\circ} \mathrm{C}$. It was taken at room temperature for samples taken into the atmosphere after deformation. The rectangular contracted sample was rubber-like above its melting point and could be elongated uniformly up to $c a$. 3.0 elongation ratio as shown in the photograph. This elongation could be done by very weak elongating force. Structural change in such an elongating process was examined by $\mathrm{X}$-ray method with the apparatus in Figure 2. 


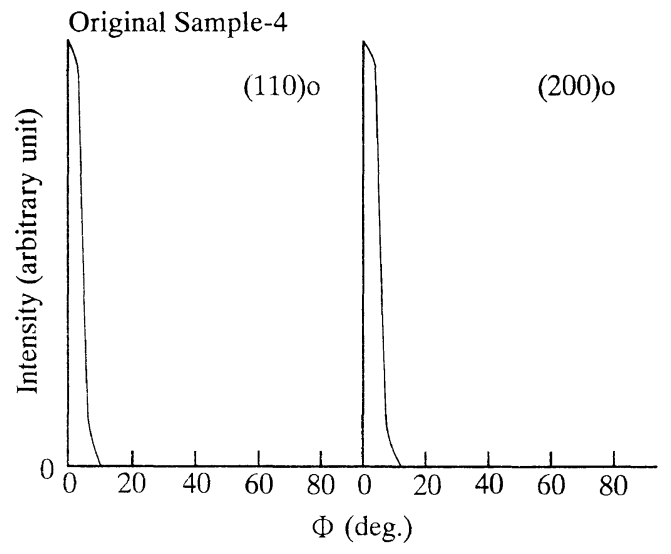

(a)

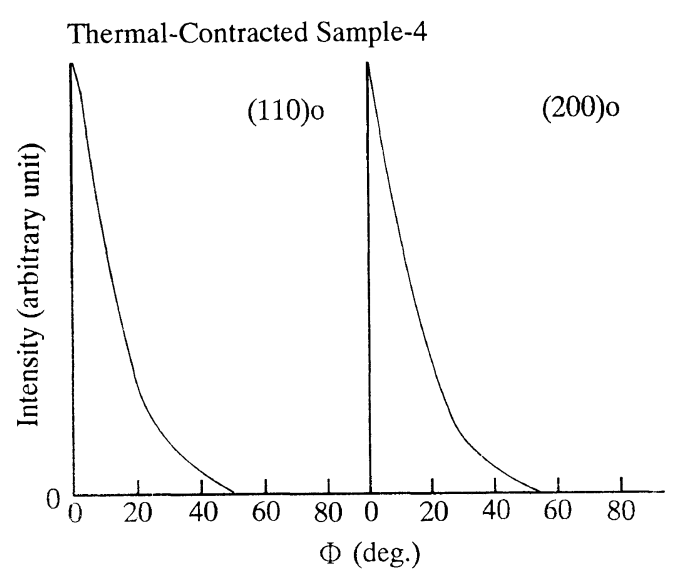

(b)

Figure 4. Azimuthal intensity distribution of (110)o and (200)o reflections for (a) original sample-4 and (b) thermal-contracted $\left(148^{\circ} \mathrm{C}\right)$ one. With the measuring system in Figure 1, data were obtained during rotation around the incident beam. The vertical scales of the (110)o and (200)o data are different from each other.

Figure 4 shows the azimuthal intensity distribution of (110)o and (200)o reflections for original sample-4 and the contracted sample. The contracted sample was obtained by contraction at $148^{\circ} \mathrm{C}$, and X-ray data were recorded at room temperature with the measuring system in Figure 1. The azimuthal angle $\Phi$ represents the angle of sample rotation from the initial one at which the fiber axis is vertical. In the case of original sample-4 (Figure $4 a)$, the data exhibit that $c$-axis of crystal is highly oriented in the direction of the fiber axis because the intensities of the two reflections decrease steeply with increasing $\Phi$. Compared to the original one, the intensities of the contracted sample (Figure $4 \mathrm{~b}$ ) decrease gradually. However, it should be noted that the crystal orientation remains appreciably in the contracted sample though the degree is lower than that in the original one.

Strain-induced crystallization was examined at three characteristic temperatures, 148,152 , and $156^{\circ} \mathrm{C}$. These temperatures were selected in the following manner. Table I is a list of DSC data of constrained sample-4, obtained by extrapolation of the peak temperatures to a heating rate of $0 \mathrm{~K} \mathrm{~min}^{-1}$. The melting and transition points determined in this way are close to quasistatic values of the constrained sample. The three elongating temperatures are all between the extrapolated melt-
Table I. Extrapolated DSC data ${ }^{\mathrm{a}}$ of constrained sample-4

\begin{tabular}{ccc}
\hline$\frac{\text { Peak } 1}{{ }^{\circ} \mathrm{C}}$ & $\frac{\text { Peak } 2}{{ }^{\circ} \mathrm{C}}$ & $\frac{\text { Peak } 3}{{ }^{\circ} \mathrm{C}}$ \\
\hline 143 & 152 & 158 \\
\hline
\end{tabular}

${ }^{a}$ The data were obtained by extrapolation of peak temperatures to a heating rate of $0 \mathrm{~K} \mathrm{~min}^{-1}$.

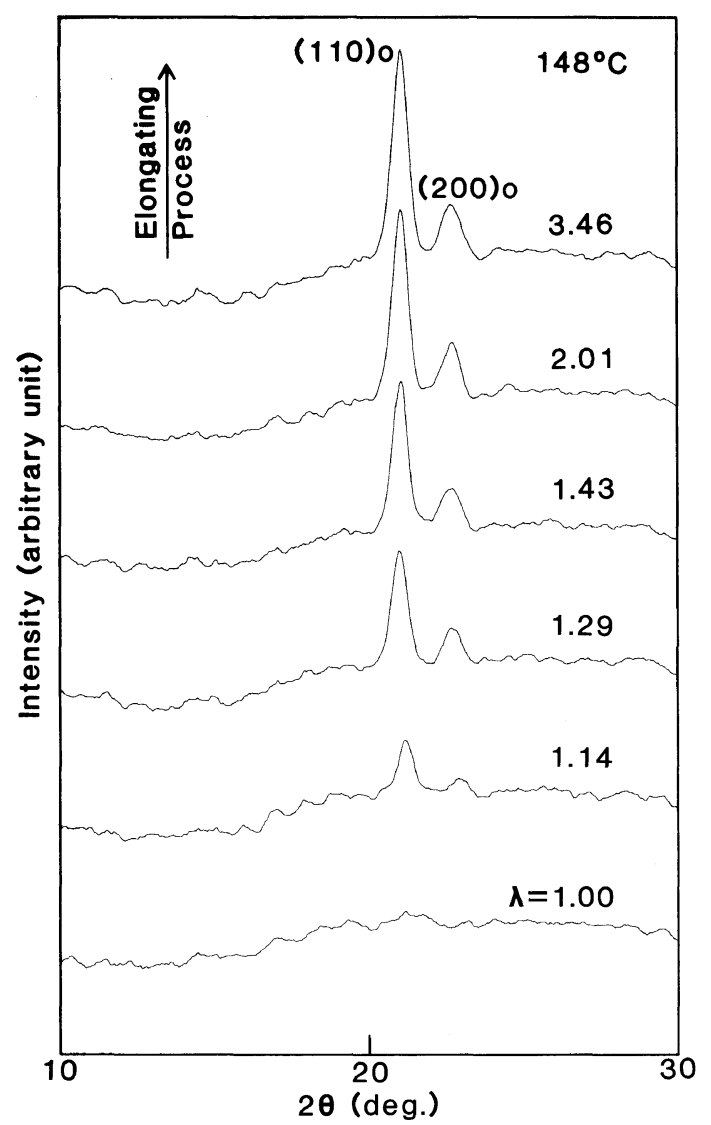

Figure 5. Change of equatorial $X$-ray diffraction pattern, measured in the elongating process at $148^{\circ} \mathrm{C}$ for contracted sample- 4 with the apparatus in Figure 2. $\lambda$ represents elongation ratio.

ing point of the unconstrained orthorhombic phase $\left(143^{\circ} \mathrm{C}\right)$ and that of the constrained hexagonal one $\left(158^{\circ} \mathrm{C}\right)$. Further, the temperature of $152^{\circ} \mathrm{C}$ is just the extrapolated transition point from the orthorhombic to the hexagonal phase, and those of 148 and $156^{\circ} \mathrm{C}$ are below and above it, respectively.

Change of the equatorial X-ray diffraction pattern, obtained in the elongating process at $148^{\circ} \mathrm{C}$, is shown in Figure 5. The patterns were measured with the apparatus in Figure 2. A small number of crystals remained without melting when the contracted sample was directly heated to the temperature. Therefore, the sample was first heated to $159^{\circ} \mathrm{C}$ and held there for 10 minutes to melt completely the remaining crystals in the only measurement at this temperature, and then cooled to $148^{\circ} \mathrm{C}$ and held there during measurement. The measurement was carried out up to the break ratio of $c a$. 3.5. Before elongation $(\lambda=1)$, there is no diffraction peak, indicating that the sample was completely melted. However, two diffraction peaks (110)o and (200)o of the orthorhombic crystals appear and become progressively greater with increasing ratio. 


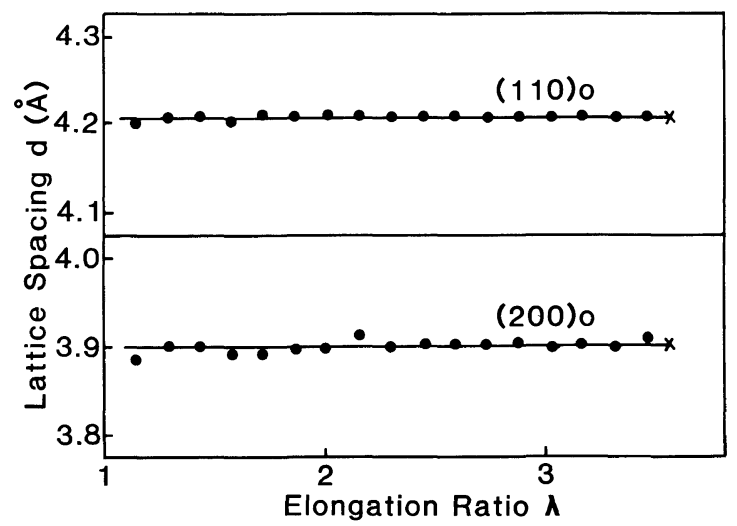

Figure 6. Change of lattice spacing $d$ in the elongating process shown in Figure 5.

This implies that orthorhombic crystals grew from the complete molten state in the elongating process, that is, strain-induced crystallization occurred. Moreover, it was confirmed that the intensities of the diffraction peaks did not change with time when the sample was maintained for long time above 3 hours at a certain ratio. For these reasons, it is concluded that the increase of the peak intensities in the figure is attributed to the increase of the crystals that grew with elongation ratio. In practice, the sample thinned approximately in inverse proportion to the elongation ratio, so that the $\mathrm{X}$-ray exposure volume decreased with it. In this work, the effect of the thinning was not corrected. If this correction is done, the increase of the peak intensities with ratio will become more significant than shown in Figure 5.

Change of lattice spacing $\mathrm{d}$ in this elongating process is shown for (110)o and (200)o reflections in Figure 6. As shown in the figure, the lattice spacing does not change up to the break ratio for both reflections, in other words, the lateral dimensions, i.e., lattice constants of $a$ - and $b$-axes did not change during the elongating process. Though the change of $c$-axis lattice constant corresponding to the fiber period was not measured in this work, it is presumed that its value did not also change during the elongating process because the elongating stress was too weak to change the value. These suggest that the unit cell of the growing crystal is not deformed by the elongating stress in this process.

Figures 7 and 8 show changes of the equatorial Xray diffraction pattern, measured in the elongating processes at 152 and $156^{\circ} \mathrm{C}$, respectively. With temperature, thermal-contractile force to the sample increases, while the tensile strength decreases. Consequently, the break ratio decreased remarkably with elongating temperature as seen in Figures 5, 7, and 8. It should be noted that the magnification ratio of the vertical scale becomes larger in the order of Figures 5, 7, and 8 . At $152^{\circ} \mathrm{C}$ (Figure 7), only a small number of crystals remained at the initial ratio $(\lambda=1)$. Therefore, the heat treatment at a higher temperature for the complete melting was not done because of an estimate that its orientation change scarcely influences the X-ray data. As shown in this figure, the intensities of the two orthorhombic peaks become larger with the elongation ratio due to the strain-induced crystallization of the orthorhombic

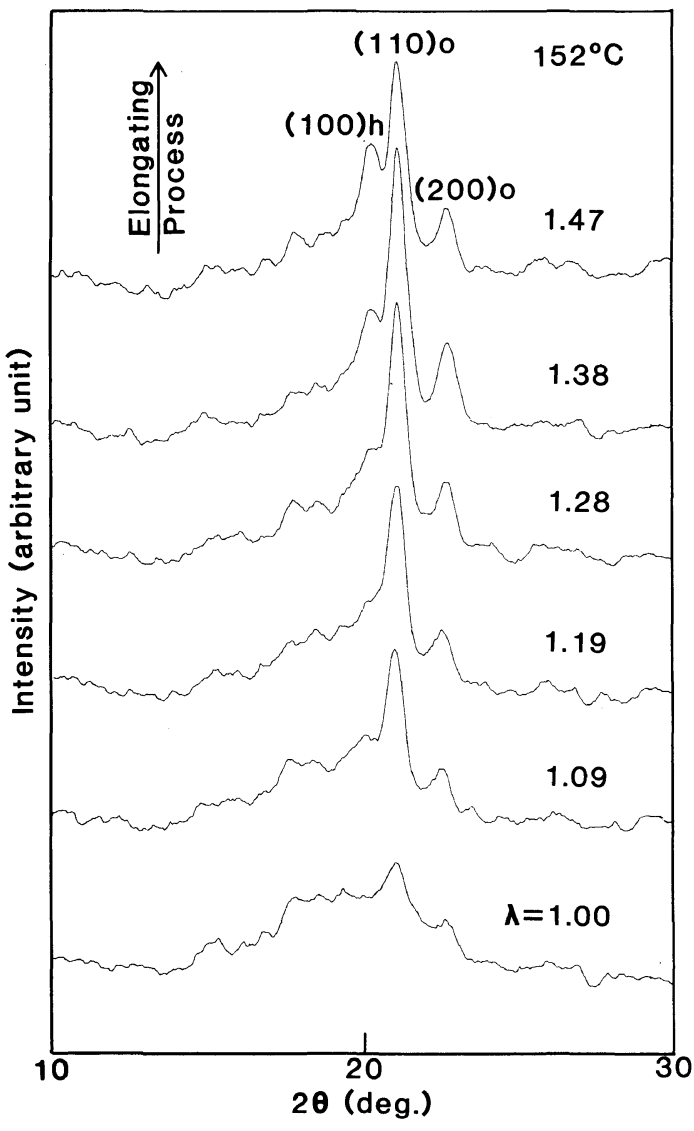

Figure 7. Change of equatorial $\mathrm{X}$-ray diffraction pattern, measured in the elongating process at $152^{\circ} \mathrm{C}$ for contracted sample- 4 with the apparatus in Figure 2.

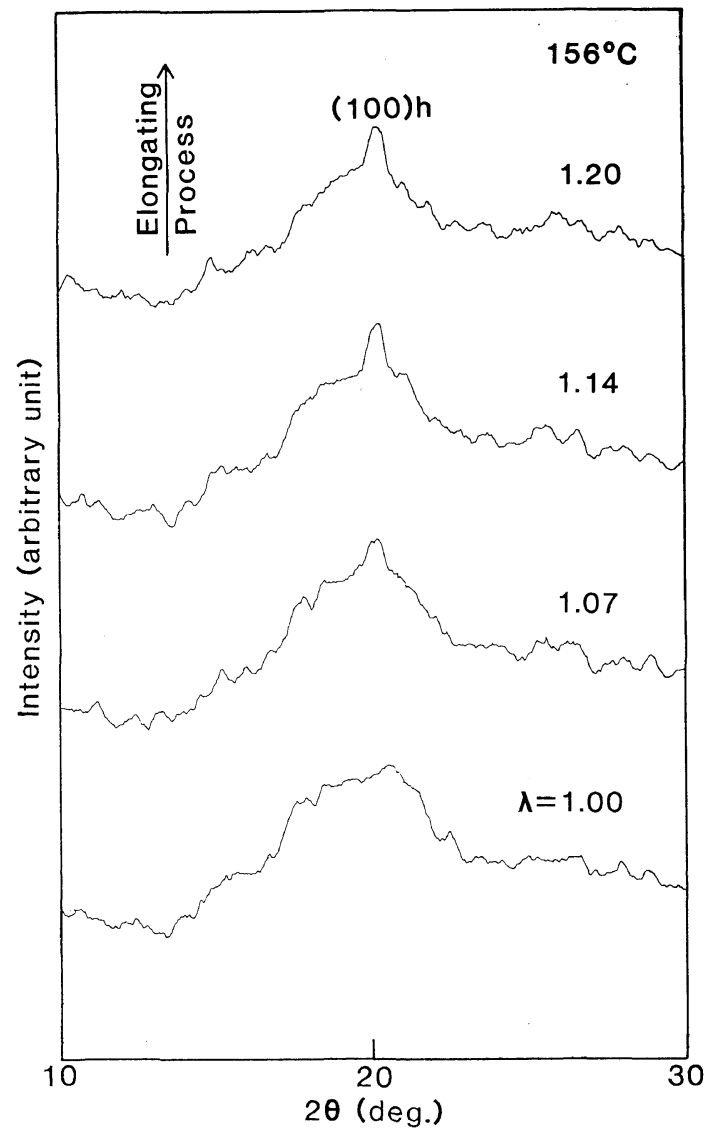

Figure 8. Change of equatorial $X$-ray diffraction pattern, measured in the elongating process at $156^{\circ} \mathrm{C}$ for contracted sample- 4 with the apparatus in Figure 2. 


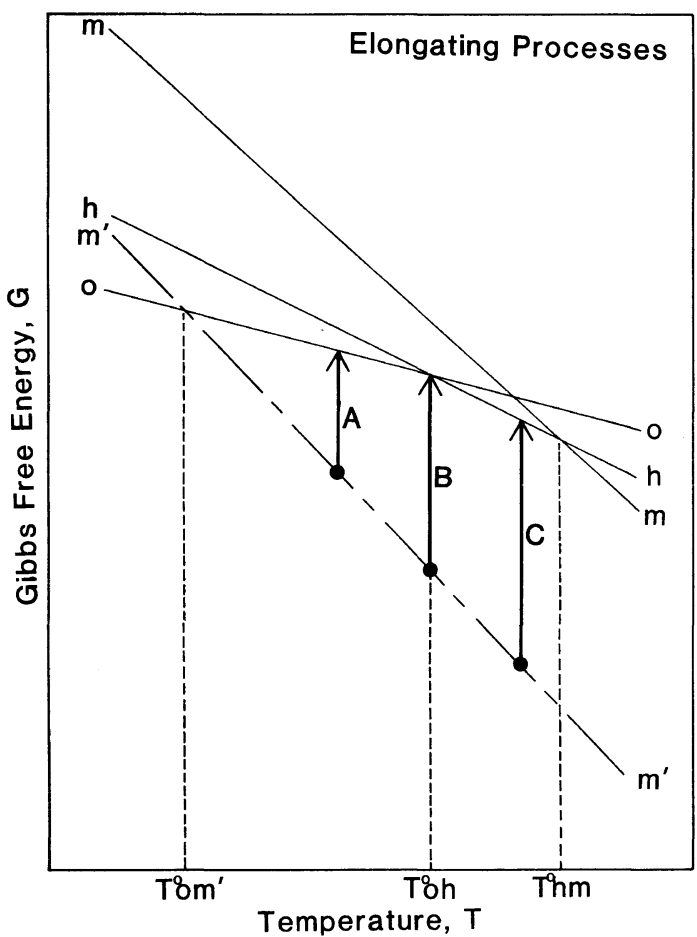

Figure 9. Schematic free energy diagram proposed in part I. Thick arrows $\mathrm{A}, \mathrm{B}$, and $\mathrm{C}$ represent the elongating processes at 148,152 , and $156^{\circ} \mathrm{C}$, respectively.

crystals. In addition, single (100)h peak of hexagonal crystal appears on the lower angle side of (110)o at elongation ratios above 1.38. This implies that the hexagonal crystals grow together with the orthorhombic ones through the strain-induced crystallization at this temperature. On the other hand, the data at $156^{\circ} \mathrm{C}$ in Figure 8 show the appearance of only weak (100)h peak, indicating that a small number of hexagonal crystals have grown by the strain-induced crystallization. As the sample broke at a small elongation ratio of 1.2 , the growth of only a few crystals could be observed at this temperature. However, it can be said that the most stable structure of the growing crystal is hexagonal since no orthorhombic peak appears.

From the results above, it is concluded that straininduced crystallization occurs during elongation of the thermal-contracted UHMW-PE above its melting point and the growing crystal structure changes from orthorhombic to hexagonal with increasing temperature. Such temperature dependence of the crystal structure formed on elongation is explained as follows on the basis of the free energy diagram shown in Figure 9. As mentioned, the elongating temperatures were selected based on the data in Table I, which give the melting and transition points close to quasistatic ones, i.e., $T_{\mathrm{om}^{\prime}}^{0}$, $T_{\mathrm{oh}}^{0}$, and $T_{\mathrm{hm}}^{0}$ of the constrained sample. That is, $148^{\circ} \mathrm{C}$ between $T_{\mathrm{om}^{\prime}}^{0}$ and $T_{\mathrm{oh}}^{0}, 152^{\circ} \mathrm{C}$ close to $T_{\mathrm{oh}}^{0}$, and $156^{\circ} \mathrm{C}$ between $T_{\mathrm{oh}}^{0}$ and $T_{\mathrm{hm}}^{0}$ were selected. In the figure, thick arrows $\mathrm{A}, \mathrm{B}$, and $\mathrm{C}$ represent the elongating processes at 148,152 , and $156^{\circ} \mathrm{C}$, respectively. At the same temperature, both the crystal growing in the isothermal elongating process and that of the constrained sample in the quasistatic melting process are presumably under very similar conditions. In other words, the elongating stress balanced with the thermal-contractile one applies to both the crystals identically. Both are expected to take the most stable structure at each temperature. Consequently, orthorhombic and hexagonal crystals that are most stable under the constrained states at $148(\mathrm{~A})$ and $156^{\circ} \mathrm{C}(\mathrm{C})$, respectively, grow in the elongating processes at the respective temperatures, and both types of crystals grow at $152^{\circ} \mathrm{C}(\mathrm{B})$, i.e., transition point $T_{\mathrm{oh}}^{0}$ at which the stabilities of both structures are identical.

In this work described in Parts I and II, melting under the constrained state and strain-induced crystallization of PE, which have not yet been studied systematically, were studied using some kinds of fibers and highly-drawn samples made from UHMW-PE, and the appearance of the hexagonal phase and temperature dependence of the growing crystal structure were shown. Further, their mechanisms were thermodynamically discussed on the basis of the free energy diagram. Such characteristic melting and crystallization processes may be observed for other fibers or highly-drawn samples made from other kinds of ultra-high molecular weight polymers, and thus the detailed studies about them are desired.

\section{REFERENCES}

1. S. Tsubakihara, A. Nakamura, and M. Yasuniwa, Polym. J., 28, 489 (1996).

2. A. Keller, J. Polym. Sci., 15, 31 (1955).

3. A. Keller and M. J. Machin, J. Macromol. Sci. Phys., B1, 41 (1967).

4. M. J. Hill and A. Keller, J. Macromol. Sci., Phys., B3, 153 (1969).

5. J. T. Judge, R. S. Stein, J. Appl. Phys., 32, 2357 (1961).

6. T. Kawai, M. Iguchi, and H. Tonami, Kolloid Z., 221, 28 (1967).

7. P. H. Lindenmeyer and S. Lustiy, J. Appl. Polym. Sci., 9, 227 (1965).

8. K. Katayama, T. Amano, T. Nakamura, Kolloid Z., 226, 125 (1968).

9. K. Kobayashi and T. Nagasawa, J. Macromol. Sci., Phys., B4, 331 (1970).

10. S. B. Clough, J. Macromol. Sci., Phys., B4,199 (1970).

11. A. Keller, Kolloid Z., 231, 386 (1969).

12. A. J. Pennings, Kolloid Z., 236, 99 (1970).

13. A. J. Pennings and A. Zwijnenburg, J. Polym. Sci., Polym. Phys. Ed., 17, 1011 (1979).

14. H. Sakami, S. Iida, and K. Sasaki, Kobunshi Ronbunshu, 34, 653 (1977).

15. S. Iida and H. Sakami, J. Chem. Soc. Jpn, 12, 1913 (1976).

16. H. Sakami and T. Ideishi, Kobunshi Ronbunshu, 36, 575 (1979). 